\title{
A decision support system for improving doctors' prescribing behavior
}

\author{
Chinho Lin ${ }^{\mathrm{a}, 1}$, Chun Mei Lin ${ }^{\mathrm{a}, \mathrm{b}}$, Binshan Lin ${ }^{\mathrm{c}, *}$, Ming-Chin Yang ${ }^{\mathrm{d}}$ \\ a Department of Industrial Management Science and Institute of Information Management, National Cheng Kung University, Taiwan \\ ${ }^{\mathrm{b}}$ Bureau of National Health Insurance, Southern Region Branch of Taiwan, Taiwan

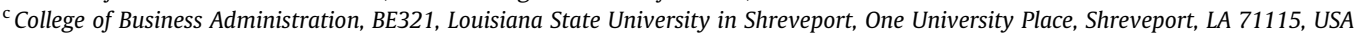 \\ ${ }^{\mathrm{d}}$ Graduate Institute of Health Care Organization Administration, College of Public Health, National Taiwan University, Taiwan
}

\section{A R T I C L E I N F O}

\section{Keywords:}

Pre-play communication

Coordination game

Prescribing behavior

Decision support system (DSS)

\begin{abstract}
A B S T R A C T
The study adopts a well-defined game theory with a decision support system (DSS) to embed the critical factors involved in coordination game equilibrium. A data-warehouse-based DSS is used as a coordinating instrument along with the disclosure of quality report cards. Feedback on prescribing, giving information on aggregated data, along with the DSS, could be sufficient to improve prescribing behavior. The aim of this study was to apply a DSS with pre-play communication of the coordination game theory to the improvement of doctors' antibiotic prescribing behavior. We examined whether the DSS improved the prescribing behavior, and assessed how well the participating doctors improved quality of care. We found the group using the system had a greater decrease in antibiotic prescription than the non-DSS group. This study concluded that the DSS with game-theory modeling has made significant contributions for improving the doctors' prescribing behavior. Future research directions and managerial implications are addressed as well.
\end{abstract}

(c) 2008 Elsevier Ltd. All rights reserved.

\section{Introduction}

Drug expenditure control is an important element in the health financial systems of all countries (Ess, Schneeweiss, \& Szucs, 2003; Okunade \& Suraratdecha, 2006). The dominant influence in prescribing drugs is the doctor and antibiotics usage has long been considered an important issue for analyzing the decision-making process in doctors' prescribing behavior (Bradley, 1991; Sintchenko \& Coiera, 2003). The development of decision support system is increasingly important in primary care for prescribing, performance measures, cost control and quality of care (Ruland \& Bakken, 2002). The supporting healthcare decision-making is dependent on the availability of integrated information and presented in a timely manner to their practice (Kim et al., 1999; Sintchenko \& Coiera, 2003; Slovensky, Fottler, \& Houser, 1998).

The quality report cards or doctor profiles extract from medical records, which reveal the difference in the quality of care delivered and prescribing information among different doctors (Hibbard, Stockared, \& Tusler, 2003). The quality report cards thus are a useful tool for peer-comparison and a voluntary response to engender trust by reassuring the public about the performance of doctors and the health care system more generally (Brownell, Roos, \& Roos, 2001). Many studies have addressed the effectiveness of public

\footnotetext{
* Corresponding author. Tel.: +1 318797 5025; fax: +318 7975127.

E-mail addresses: linn@mail.ncku.edu.tw (C. Lin), Binshan.Lin@LSUS.EDU (B. Lin).

1 Tel.: +886 6 2757575x53137; fax: +886 62759451.
}

quality reports in stimulating hospital quality improvement, costs control and supporting patient choice (Hibbard, Stockared, \& Tusler, 2005; Mason \& Street, 2005). At the same time, a third-party insurance payer can monitor a doctors' adherence to prescribing guidelines and measure the prescription rate being provided under doctor profiles (Maryniuk, 1990). Nevertheless, this disclosure process may encourage health care providers to play "games" within the delivery of health care by changing their patterns of practice (Dranove, Kessler, McClellan, \& Satterthwaite, 2003). This phenomenon usually occurs when quality report cards and payment-auditing (payoff) measures are directly related (Chinburapa et al., 1993; McNamara, 2006; O’Brien, 1988).

The doctor's prescribing behavior change with payment auditing process can be modeled by the coordination game when the third-party insurance payer audits the doctors' claim procedures (Burton \& Sefton, 2004; Dixit \& Skeath, 1999). One feature of a coordination game is that the conditional best response involves a matching of strategies across players (Alm \& McKee, 2004). In particular, costless signaling in coordination games has the potential to increase the possibility that the efficient equilibrium will be reached by players (Camerer, 2003). Hence the available information that allows doctors to focus on a particular strategy, with costless signaling by disclosing the information in quality report cards, also called "pre-play communication", by which the doctors' prescribing behavior assessment will be delivered by comparing their own performance with that of their peers. Moreover, a pre-play communication that mimics the information provided by the 
quality report cards disclosure allows doctors to coordinate antibiotic prescriptions under the claim auditing rules.

The study developed a data-warehouse-based decision support system (DSS) that displays the quality report cards and allows physicians to search for their own percentage of antibiotic prescriptions as well as compare those results with others. The aim of this study was to apply the DSS with pre-play communication of the coordination game theory to the improvement of doctors' prescribing behavior. This application via the DSS discloses the percentage of antibiotic prescriptions in the quality report cards of doctors and their peers as pre-game communication. The pre-play communication paradigm will also implicitly affect and reshape doctors' practice patterns, which will be conducted under the influence of peer-comparison, and the doctor will therefore make more appropriate decisions concerning utilization of antibiotics (Lambert et al., 1997).

It will then be useful to investigate whether the doctors in prescription integrate such information into their decision-making processes in order to obtain the optimal outcome and avoid refusing reimbursement under the medical claims payment audit (MCPA) procedures. The study also helps policy makers to understand the extent to which the quality report card can be a coordinating instrument to facilitate the coordination of practice patterns among doctors. Furthermore, it allows the doctors and the Taiwan's Bureau of National Health Insurance (TBNHI) to obtain consistency in reaching win-win situations when dealing with prescribing patterns and cost containment.

The remainder of the paper is organized as follows. Section 2 provides the underlying theory of reimbursement of health care with the policy of medical claim payment auditing (MCPA) procedures, decision support systems, and coordination games. Section 3 depicts the framework of study, DSS design and data collection. Section 4 demonstrates the results of the system evaluation. In Section 5 we present the computational results. Finally, we present concluding remarks and suggestions for future research in Section 6.

\section{Literature review}

In this section, we briefly review the related concepts about the medical claim payment auditing (MCPA) procedures, the technology in the data warehouse decision support systems and the underlying theory of coordination games.

\subsection{The medical claim payment auditing (MCPA) procedures before reimbursement}

Taiwan has implemented national health insurance from 1995. The Taiwan's Bureau National Health Insurance (TBNHI), with single-payer health insurance systems, governs drug costs and regulates drugs payment list and indication for treating infections. Many studies argued a doctors' prescription is influenced by health insurance and health-related policies (Alvanzo, Cohen, \& Nettleman, 2003; Chiang, 1997). In particular, the payment of Taiwan's National Health Insurance (NHI) based on a fee-for-service payment schedule has led to a higher volume of prescriptions from physicians (Cheng, 2003). The third-party insurance payer therefore needs to adopt an array of strategies to combat this problem. Both utilization review and physician profile analyses have been a critical factor in controlling health care costs and assurance quality of care after implementing NHI in Taiwan (Cheng, 2003; Chiang, 1997).

The medical claim payment auditing (MCPA) strategy is a major action against doctors who deviate from the prescribing guidelines or TBNHI's regulations (Fu, Tsai, Lin, \& Wei, 2004). The effective
MCPA procedures, which operate by thorough peer review activities to eliminate any charges that violate the MCPA rules, are a key tool in reducing a physician's inappropriate prescriptions and controlling health expenditures (Denig, Haaijer-Ruskamp, \& Zijsling, 1988; Hellinger, 1996).

With the MCPA procedures, monthly medical claims reimbursement is examined by the TBNHI. The TBNHI determines the doctors' medical claim reimbursement based on the audit rules concerning billing claims and tries to determine whether the medical claim needs to undergo the medical records peer-review procedures before reimbursement is made. Therefore, doctors should aim to not violate any of the rules and policies established by the TBNHI (Cheng, 2003). In general, the audit rules summarize information concerning medical claim billing (e.g. the average claim price, the amount of medical claim fee) and quality (e.g. the percentage of antibiotics, the percentage of injections). These rules are derived from the doctors' profiles by averaging the quantity contents of the doctors' cohort, which results in the discriminate indicators of claim billing and patterns of prescribing, called "auditing flags" that decide whether the doctors' claims need to undergo the medical records peer-review procedures.

Hence, a doctors' probability of medical records peer-review procedures is based not only upon his/her patterns of prescribing, but also upon these patterns of prescribing relative to his/her peers. Thus, doctors have to consider the behavior of others, and then follow a similar course of action in order to avoid being subject to the medical records peer-review procedures. Therefore, a basic rule for doctors' patterns of prescribing is "not significantly different from your peers" (Fu et al., 2004). Nevertheless, the medical records peer- review activities are time-consuming and raise personnel costs, so under budget constraints the TBNHI can only audit those claims that are higher than the MCPA threshold. Thus, there is a game among physicians and between physicians and TBNHI. In order to increase the doctors' compliance on reimbursement regulations, the TBNHI announces the MCPA rules to doctors before claims are submitted. As a result, the MCPA rules guide the doctors' prescribing patterns to approach an equilibrium state under the MCPA's threshold (Alm \& McKee, 2004; Dixit \& Skeath, 1999). The coordination game can be employed under the auditing process and thus change these doctors' prescribing behavior (Cooper et al., 1990, 1992; Shiell \& Chapman, 2000; Van Huyck, Battalio, \& Beil, 1990).

\subsection{Data-warehouse-based decision support system}

As health information technology continues to improve, electronic medical records are now kept in digital form and stored in data warehouses, and facilitate developing decision support system to be a direct aid on clinical decision-making to increase health care quality and efficiency (Lovell \& Celler, 1999; Short, Frischer, \& Bashford, 2004; Tsai \& Kuo, 2007). Data warehouses have emerged to meet this need in healthcare. A data warehouse, as a repository for data extracted from various operational health information systems, facilities the third-party insurance payer to audit electronic medical records and transaction processes to ensure heath services are more accountable (March \& Hevner, 2007; Watson, Fuller, \& Ariyachandra, 2004). Data warehousing offers benefits such as cost savings and error reduction from the consolidation of multiple, disparate decision support platforms in clinical practice. It can be used to support queries, reporting, online analytical processing (OLAP) and data mining for all levels of management decision-making processes (Kim, Hong, \& Park, 2008). A data warehouse is also at the heart of decision-making in the clinical practice, which integrates data from disparate sources into one coherent repository of information to give a better picture of the overall situation ((March \& Hevner, 2007). Such systems have be- 
come central for decision support in health care (Berndt, Hevner, \& Studnicki, 2003; Pedersen \& Larsen, 2001), and provide the data infrastructure that is needed for decision support (Ruland \& Bakken, 2002; Watson et al., 2004).

Decision support systems (DSS) is an interactive, computerbased system intended to provide support to decision-makers engaged in solving various semi- to ill-structured problems involving multiple attributes, objectives and goals (Nemati, Steiger, Iyer, \& Herschel, 2002). Integrating the data warehousing technology can aid decision-makers and physicians in health care to make appropriate and cost-effective decisions under the cost containment policy (Liang, Xue, \& Berger, 2006). DSS is thus becoming increasingly critical in the daily operation of health care (Ruland \& Bakken, 2002), with physicians using DSS to make strategic decisions and improve their clinical operations and quality of care. However, DSS is dependent upon knowledge acquisition for the design of the decision mechanism in terms of (1) the clinical domain, for understanding of the decision problem; (2) decision science and research on practical decision-making motivation; and (3) informatics technology, to provide the technology and algorithms for the data collection, processing, structure, presentation and integration of the database (Nemati et al., 2002; Pedersen \& Larsen, 2001; Ruland \& Bakken, 2002).

Accordingly, using the quality report cards as a pre-play communication is proposed to show how the DSS is an important instrument in the coordinating process among doctors and the $\mathrm{NHI}$ auditing policy. It is here that the notion of a "coordination game" enters in the design of the decision support systems. The DSS provides a close to the time of information to doctors so that they can analyze the probability of auditing and make more advantageous decisions after comparing their antibiotic prescription rates with their peers.

\subsection{Coordination game}

Game theory is one of the most commonly used tools for modeling and analyzing management problems, which are characterized by both multi-period runs and strategic decision-making. Game theory techniques have also been widely employed to deal with issues in health care management (O'Brien, 1988; Shiell \& Chapman, 2000), and physicians and third-party insurance payers can thus be viewed as the players engaged in a game (Ford, Wells, \& Bailey, 2004). Doctors are the ones who realize both the interdependence of individual practice strategies and the health insurance policies or regulations that are the basic criteria of the game. The coordination game can create agreements among the players about their preferences among the possible outcomes (Colman, 1995). The coordination game is defined by four elements (Rasmusen, 1994):

(1) The set, $i=\{1,2, \ldots, n\}$, of players.

(2) The sequence, $s=\left\{s_{1}, s_{2}, \ldots, s_{n}\right\}$, of strategy sets of the players.

(3) The sequence, $\pi_{i}\left(s_{1}, \ldots, s_{n}\right)$, of payoff functions of the players, and

(4) The sequence, $F:\left\{s_{1}, \ldots, s_{n}, \pi_{1}, \ldots, \pi_{n}\right\} \rightarrow s^{*}$ is a rule that defines an equilibrium and is based on the possible strategy combinations and the payoff functions.

The coordination game equilibrium, $\forall i, \pi_{i}\left(s_{i}^{*}, s_{-i}^{*}\right) \geqslant \pi_{i}\left(s_{i}^{\prime}, s_{i}^{*}\right), \forall s_{i}^{\prime}$, and the strategy combination $s^{*}$ is a Nash equilibrium if no player has an incentive to deviate from their strategy since other players do not deviate. A rational player is judicious if they have a clear preference, which is represented by a game function payoff that can be expressed in terms of utility, profit, sales revenue, negative cost, or any other such quantitative measure (Dixit \& Skeath,
1999). There is a high degree of success in attaining the payoff dominant equilibrium outcome in a coordination game, since the best outcome is that considered best by the other players.

More than one equilibrium strategy is usually available in this type of game, and the player makes decisions in a way consistent with their objective, namely, to maximize their payoff in the game. Players will have the highest payoffs if they can coordinate a strategy when they play the game (Cooper et al., 1992). This is the basic concept of focal point equilibrium, where players play their best response because of its differential characteristic (Schelling, 1960). In other words, if the players have information that enables them to focus on a particular strategy, then the likelihood of reaching the coordinated result will be enhanced (Alm \& McKee, 2004). The best response is the basic criteria in game playing that involves matching strategies across players, and thus a coordination game cannot reach its dominant strategy. Often there is an efficient outcome, which can only be achieved through coordination. When players communicate, the desire to obtain optimal results will encourage them to collaborate.

Many studies have found that pre-play communication (i.e. cheap talk) is quite effective in achieving Pareto-efficient outcomes when players coordinate (Dixit \& Skeath, 1999; Schelling, 1960). Cooper and et al. (1990) found that a high degree of success can be attained when there is a payoff-dominant equilibrium outcome in a coordinated game. One-way pre-game communication could also be quite effective in achieving coordination relative to the no-communication baseline in games with a cooperative strategy (Cooper et al., 1992). Farrell (1993) argued that cheap talk is a signaling game in which neither player 1's nor player 2's payoff is dependent on the message. The ability to communicate often affects the outcome of strategy interaction. Burton and Sefton (2004) found that when players have an opportunity to do pre-play communication the possibility of equilibrium will increase significantly and communication plays a key role in reassuring the subjects that certain strategies will be used in the game, allowing them to coordinate and create an efficient equilibrium. As a result, pre-play communication enables players to coordinate and reach the focal point equilibrium.

\section{Empirical study}

In this section, we illustrate how a DSS as a pre-play communication plays an important role in the coordination process. We have designed a data-warehouse-based DSS that employs technology to gather and analyze a large quantity of information in this study.

\subsection{Conceptual framework}

We assume that the doctors' prescribing behavior change with payment auditing process is a coordination game, and the thirdparty insurance payer offers a DSS to disclose the peer-comparison information for increasing Nash equilibrium. The coordination game equilibrium is $\forall i, \pi_{i}\left(s_{i}^{*}, s_{-i}^{*}\right) \geqslant \pi_{i}\left(s_{i}^{\prime}, s_{-i}^{*}\right), \forall s_{i}^{\prime}$, where $i=\{1,2$, $\ldots, n\}$ is a set of doctors, where $s=\left\{s_{1}, s_{2}, \ldots, s_{n}\right\}$ is a set of prescribing strategy, where $\pi_{i}\left(s_{1}, \ldots, s_{n}\right)$ is a set of payoff function under prescription rate and MCPA procedures, where $F:\left\{s_{1}, \ldots s_{n}\right.$, $\left.\pi_{1}, \ldots, \pi_{n}\right\} \rightarrow s^{*}$ is a set of equilibrium based on the appropriate the strategy and payoff function.

The effectiveness of the game is measured by how much the circumstances surrounding decision-making can be stimulated. This can be separated into five stages (Elgood, 1984):

1. Determination of objectives and/or identification of problem.

2. Collection and/or analysis of information.

3. Definition and/or comparison of an alternative course of action. 
4. Action.

5. Review of results (feedback on payoff).

Among the five stages, it has been widely recognized that when doctors are given pre-play communication from quality report cards and allow a majority of doctors to learn the optimal strategies to test conditional rules, which increases the likelihood of a coordinated outcome or Pareto-efficient outcome (Brandts \& Charness, 2003; Burton \& Sefton, 2004; Cooper et al., 1992; Dixit \& Skeath, 1999; Jaegher \& Jegers, 2001; Schelling, 1960). The coordination game allows a doctor (player) to predict the most likely course of action that will be taken by each doctor based on his/ her knowledge of the available options and his/her expected payment under the auditing policy. Hence a payment audit game among doctors is used to ensure that their patterns of prescribing antibiotics do not deviate too much from the average of their peers. When a doctor contemplates the payment (or payoff) he/ she will have an incentive to coordinate changes in antibiotic prescriptions.

The optimal practice for each doctor is to coordinate on submitting zero antibiotic prescriptions, because this yields the highest payoff when the TBNHI only audits on the basis of the difference. However, the MCPA procedures are an infinite game when the doctors make a continuous contract with TBNHI. If a doctor decides to submit zero antibiotic prescriptions it will create a narrow strategy space in the future (i.e. a loss of drug payment on reimbursement). Hence, the doctors need to consider their peers antibiotic prescriptions in order to make appropriate decisions. Accordingly, it is worth exploring the connection between the coordinating instrument (DSS discoursing quality report cards) and the changes in doctors' prescription patterns. A conceptual framework of the doctors' prescribing decision-result cycle is used as the basis of this study, as depicted in Fig. 1.

A rational doctor coordinates their patterns of prescribing on an equilibrium via the DSS (revelation of quality report cards) coordinating device to find the difference between their antibiotic prescriptions and those of their peers. The doctor will then their patterns of prescribing accordingly (Ahluwalia, Weisenberger, Bernard, \& McNagny, 1996). DSS is a useful coordinating instrument to aid doctors in predicting the probability of MCPA action being taken. Although the DSS may substantially change a doctors' prescribing behavior of prescribing antibiotics, the extent of this change is also dependent on doctors' own monetary payoff. Hence, we supplement the MCPA procedures to be conducted on the basis of deviations of prescription patterns. Under the MCPA procedures there are thorough peer review activities for ensuring that any prescription provision complies with clinical guidelines and TBNHI regulations, and the cases that violate the guidelines and regulations will be refused reimbursement from the MCPA procedures. This monetary payoff is central part of the doctors' prescribing decision (Dixit \& Skeath, 1999).

\subsection{Decision support system design and architecture}

A DSS is used as the coordinating device for the disclosure of quality report cards in this study based on previous research into a form of pre-game communication that facilitates coordinating. This is illustrated in Fig. 2. Antibiotic prescriptions for outpatients are chosen as the quality report cards, which are the average percentage of antibiotics prescribed per outpatient visit. The DSS's scheme consists of three components: (1) aggregating the differential claim dataset into the data warehouse; (2) OLAP technology, which integrates the functionality of earlier generations of data warehouse information technology and changes the data into multidimensional cubes that can be observed faster from all perspectives and produce varied quality report cards (Tremblay, Fuller, Berndt, \& Studnicki, 2007); and (3) a Web-based interface that provides a convenient channel for a doctor to use ( Bhargava, Power, \& Sun, 2007; Kruck, Teer, \& Christian, 2008).

The aggregated doctors' claim tables are obtained from patient service list table and patient service content table. The doctors' claim payment tables contain 25 attributes, which correspond to doctors' service provision. We use on-line analysis processing (OLAP) (Microsoft OLAP services, Taiwan) to extract the necessary data from the data warehouse for building the multidimensional cubes, which consist of the doctors' information from all perspectives (e.g. per patient, per visit antibiotic usage) (Berndt et al., 2003). The aggregate prescriptions data warehouse is then calculated based on the data extracted above. Next, comparing a doctor's prescription rate of antibiotics with all other doctors' norms derives the percentile range of the indicators. Finally, the system assigns individual codes and passwords for integrating the results of individual quality report cards. The data are placed on the Webbased interface DSS (Lee \& Chung, 2005). The doctors can search for their own percentage of antibiotic prescriptions and make comparisons with percentile distributions (see Figs. 3 and 4). The system records when the doctors access the database, and then are stored in a log database.

Figs. 3 and 4 show that when doctors use the Internet to search the quality report cards, different colors light up to inform users what percentile of antibiotic prescriptions belongs to which group. Camerer (2003) pointed out that traffic lights are familiar examples of a "correlating device" - a public, observable signal from which doctors can adjust their strategy according to their circumstances. The colors used follow those of traffic lights: red means that the antibiotic prescriptions exceed the 90th percentile, yellow lies between the 90th percentile and 75th percentile, and green means that antibiotic prescriptions are below the 75th percentile. The red light tells the doctors that they fall within an extreme group when compared with their peers. As a result, the doctor needs to decrease their antibiotic prescriptions. Otherwise they will be subjected to MCPA procedures. The doctors must keep their antibiotic prescriptions below the 75th percentile, or in the "green light", in

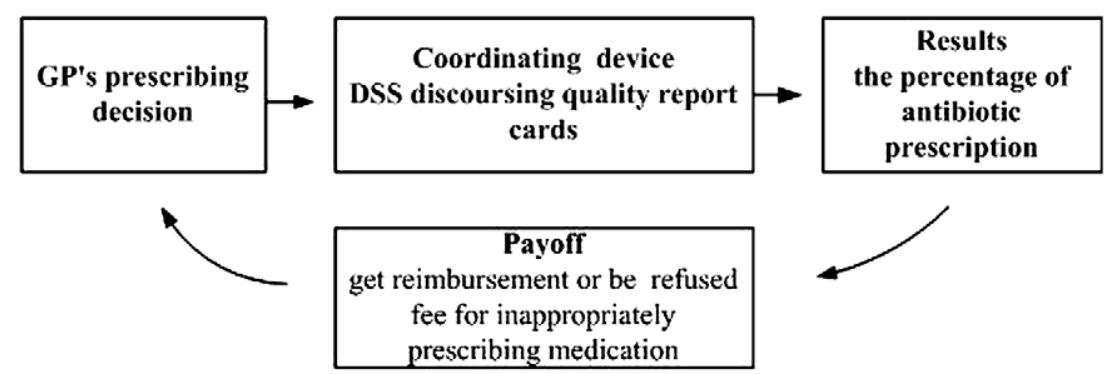

Fig. 1. The cycle of doctors' prescribing decision. 


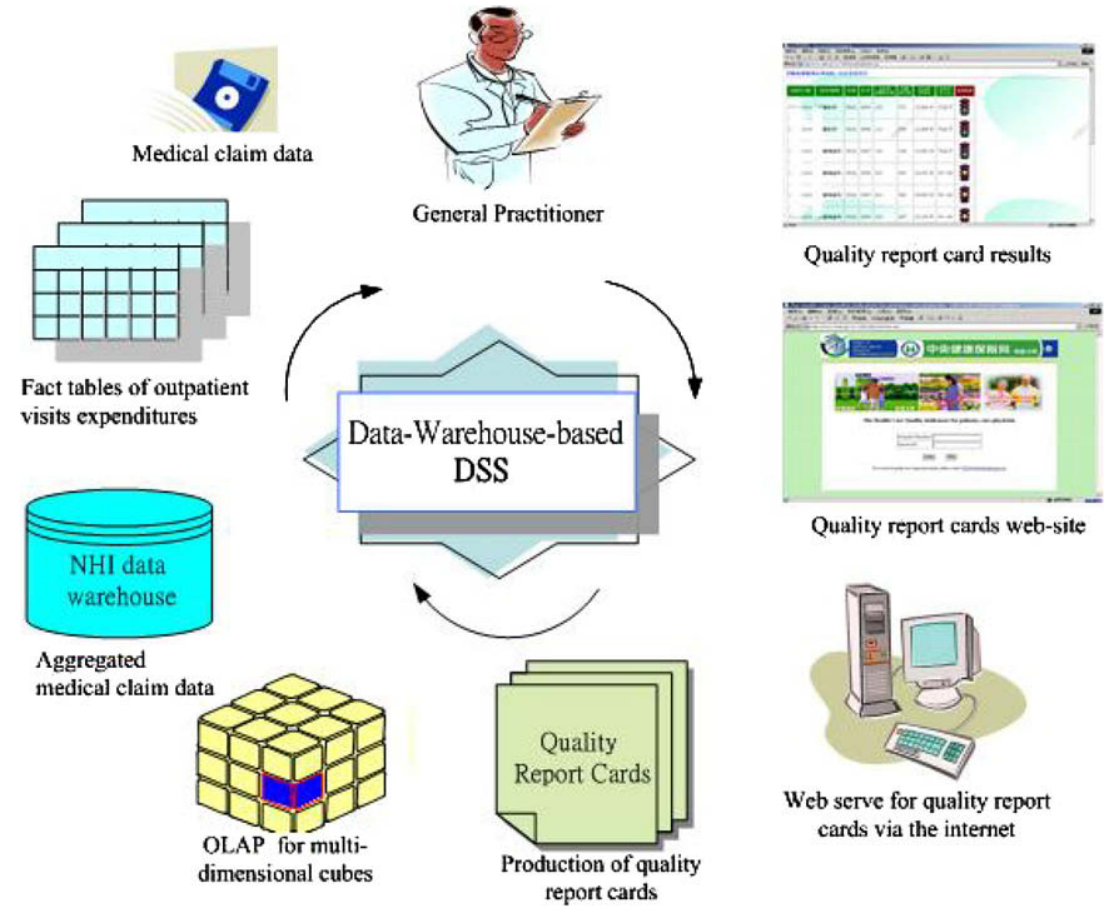

Fig. 2. Doctors' medical claim profiles gathered and the procedure for creation of DSS quality report cards.

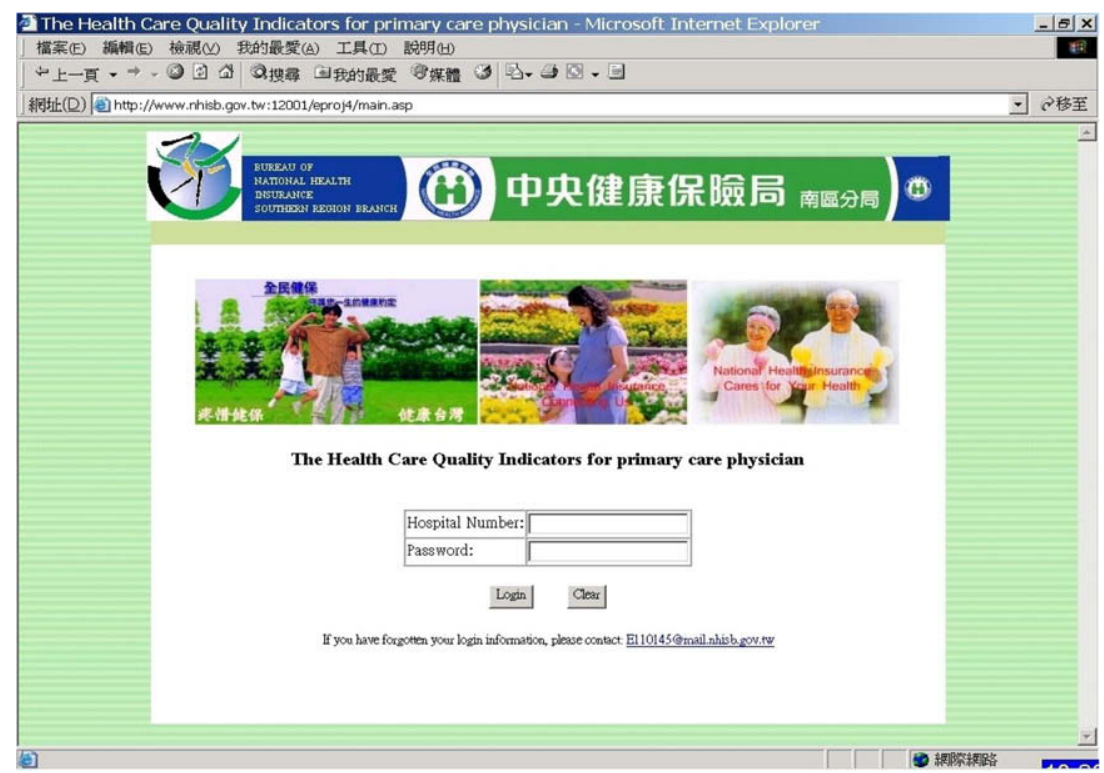

Fig. 3. An example of a DSS quality report card on the TBNHI Website.

order to prevent medical peer-review procedures by the MCPA. Therefore, the correlating instruments are useful in triggering collective action when explicit communication is cumbersome or prohibited. Thus, we adopt an auxiliary "traffic light" to disclose quality report cards on the DSS.

\subsection{Data collection}

During the research for this study we contacted a total of 1457 doctors who had contracts with the TBNHI, with practice areas covering two cities and three counties of TBNHI's southern branch.
Overall, there were 3.6 million beneficiaries receiving primary care from the doctors. Prescriptions were collected for all outpatient visits and came from the computerized claims data warehouse of the NHI, so that we could evaluate the percentage of antibiotic prescriptions among the contracted doctors. The medical claims data warehouse contains data for a two-year time period. On average, the system handled over 2.7 million cases involving outpatient patient visits services every month.

This natural experiment was uncontrolled for before and after comparisons. Use of the DSS was voluntary though strongly encouraged. During this period, the TBNHI periodically offered 


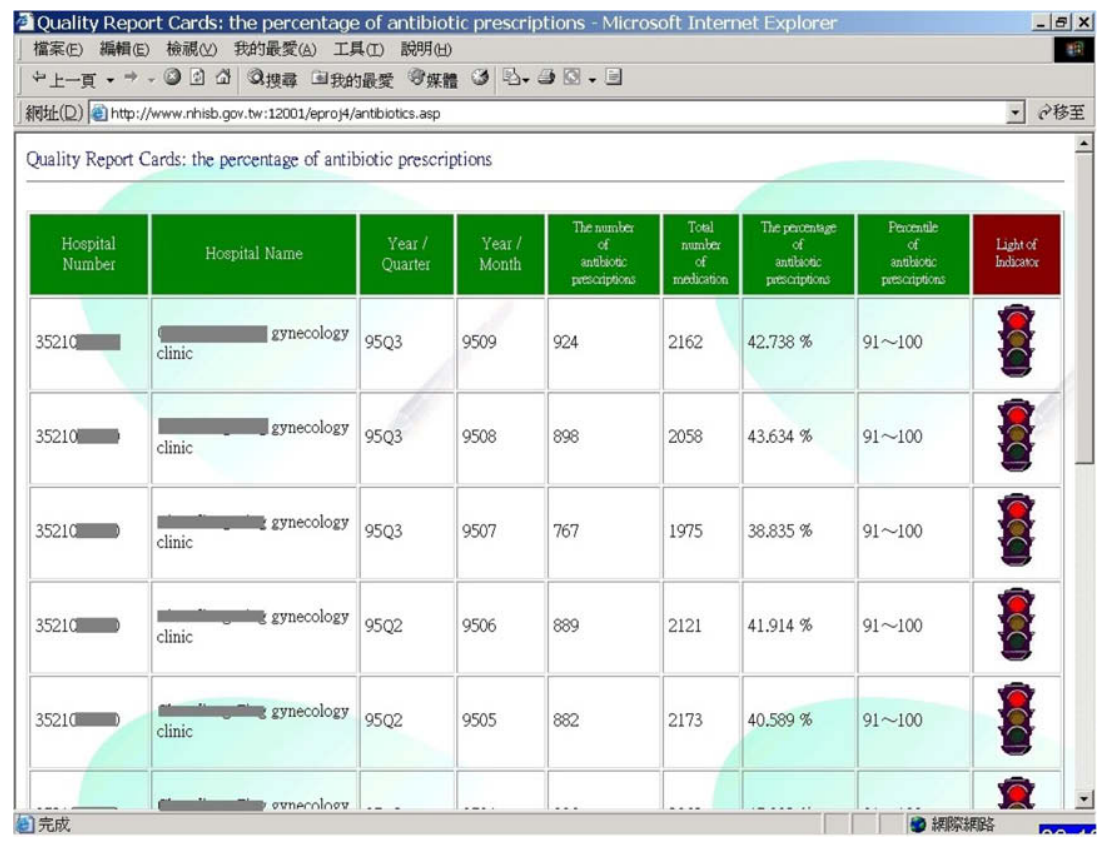

Fig. 4. The query results from DSS.

evidence-based medical guidelines and education in antibiotic prescriptions to doctors with whom it had a contract. There were 1102 doctors $(87 \%)$ in the education only group (non-DSS group) and 164 doctors (13\%) in both the education and using DSS group (hereafter DSS group). Among the 1457 doctors, 191 doctors were not included in the analysis because their data was incomplete during the study period, due to the doctors' withdrawal from the TBNHI contract. Therefore, the final analysis was based on 1266 doctors and consisted of 1102 doctors in the non-DSS group and 164 doctors in the DSS group. The baseline quarter and the postintervention percentages of antibiotic prescriptions were taken from TBNHI's data warehouse, which has collected medical claims for all outpatient-visits.

This two-year study was divided into five quarters by each quarter after the baseline quarter. The model, which uses the coordinating instrument and doctors' prescription rate change when prescribing antibiotics, based on the following three assumptions:

1. A doctor who suffers from over prescribing antibiotics is described by a loss function. It shows the relationship between prescribing antibiotics and the higher probability of underlying medical records peer-reviews procedures by the MCPA.

2. Operating in a consumer society, doctors tend to improve the quality of care, obeying the guidelines for antibiotic prescriptions.

3. Each doctor faces a population of patients who may suffer from either a high severity or a low severity illness, both with the same probability. Therefore, the probability of antibiotics usage is the same.

We compared the changes in the percentage of antibiotic prescriptions for all. The percentage of antibiotic prescriptions in outpatient visits between the DSS and non-DSS group. Both $X^{2}$-test and $t$-test were used to analyze significant differences. Statistical analysis was conducted using the Statistical Package for the Social Sciences (SPSS 15.0 for Windows, 2006, SPSS, Chicago, IL). The results of the analysis will be discussed in detail in the following sections.

\section{Results}

In this section, we describe the results of the statistical analysis and incorporate the DSS using group feedback on the percentage of antibiotic prescriptions gathered. We also compare the variations in percentiles of antibiotic prescriptions across the two groups in the five quarters.

\subsection{Description of the sample}

The average doctors medical claim fee per month was $18,502 \pm 14,632$ (\$UD). The doctor's average age was $49 \pm 11$ years. The percentages of antibiotic prescriptions from the baseline quarter to quarter $\mathrm{V}$ were $17.3 \%, 16.1 \%, 16.0 \%, 15.7 \%, 15.3 \%$, $14.3 \%$, respectively. The results are presented in Table 1 . The TBNHI's southern region branch used educational lessons and quality report cards to decrease the percentage of antibiotics prescription. This caused the percentage of antibiotic prescriptions for outpatient visits to drop from $17.3 \%$ to $14.3 \%$.

Table 2 shows the differences in the doctors' antibiotic prescriptions rate between the non-DSS and DSS group $(p<0.001)$. The results of the analysis indicate that younger doctors and those with higher claim fee per month are more likely to use DSS.

Table 1

Percentage of antibiotic prescriptions at different quarters and doctors' characteristics $(n=1266)$.

\begin{tabular}{lll}
\hline Criteria & Mean & SD \\
\hline Baseline quarter & 0.1729 & 0.1752 \\
Quarter I & 0.1613 & 0.1670 \\
Quarter II & 0.1604 & 0.1684 \\
Quarter III & 0.1565 & 0.1593 \\
Quarter IV & 0.1526 & 0.1544 \\
Quarter V & 0.1428 & 0.1548 \\
Monthly amount of medical claim fee (SUSD) & 18502 & 14632 \\
Age (year) & 49 & 11 \\
\hline
\end{tabular}


Table 2

The relationship of doctors' characteristics with the non-DSS group and DSS group ( $n=1266)$.

\begin{tabular}{|c|c|c|c|c|c|c|}
\hline \multirow[t]{2}{*}{ Criteria } & \multicolumn{2}{|c|}{ Non-DSS group $(n=1102)$} & \multicolumn{2}{|c|}{ DSS group $(n=164)$} & \multirow[t]{2}{*}{$t$} & \multirow[t]{2}{*}{$p$-Value } \\
\hline & Mean & SD & Mean & SD & & \\
\hline Age (year) & 50 & 11 & 46 & 9 & -4.375 & 0.000 \\
\hline A amount of medical claim fee (\$USD) per month & 17,397 & 12,289 & 25,931 & 24,035 & 6.024 & 0.000 \\
\hline
\end{tabular}

\subsection{Inter-group validity analysis at the baseline quarter}

The percentage of antibiotic prescriptions distribution is skewed to the right. We use the percentiles range to compare individual observation with a set of norms to replace the geometric mean. Then we divide all of the doctors into one of the following three groups: the lower group is less than the 50th percentile, the middle group lies between the 50th and 75th percentile, and the higher group is greater than the 75th percentile. Table 3 shows the percentile distribution for antibiotic prescriptions.

Since all of doctors' prescriptions behavioral changes are influenced by MCPA's procedures or the use of DSS, it is necessary to examine the differences between the non-DSS group and DSS group within the MCPA procedures and the three percentile groups at the baseline quarter. Based on the Chi-square analysis, we found that there was no variation in the distribution of doctors between the non-DSS group and DSS group in the three percentile groups of antibiotic prescriptions $\left(X^{2}=3.06 ; p>0.01\right)$. There was also no variation for the MCPA procedures $\left(X^{2}=0.6684 ; p>0.01\right)$. This statistical analysis indicates that there are no significant differences between the DSS and non-DSS groups at the baseline quarter. This adds validity to using DSS or non-using-DSS groups as our unit of analysis in the different quarters (see Table 4).

4.3. Changes in the percentage of antibiotic prescriptions between the DSS group and non-DSS group at the different quarters

We use the $t$-test to exam the relationship between the non-DSS group and DSS group, and the percentage of antibiotic prescriptions at the five quarters. Before examining the variation in the percentage of antibiotic prescriptions between the non-DSS group and DSS group for the three percentile groups, the $t$-test for equality of mean is used to show that the differences between the variances are not significant at $5 \%$.

Table 3

The percentile distribution of antibiotic prescriptions.

\begin{tabular}{ll}
\hline Percentile criteria & Percentage of antibiotic prescriptions \\
\hline 25th Percentile & 0.034 \\
50th Percentile & 0.107 \\
75th Percentile & 0.271 \\
90th Percentile & 0.431 \\
\hline
\end{tabular}

There was no statistical significance $(p>0.01)$ in the lower and middle percentile groups during the five quarters between the non-DSS group and DSS group as shown in Tables 5 and 6. Table 7 presents the results of the higher group, where there are statistically significant declines in the percentage of antibiotic prescriptions for the five quarters. This difference declined as time increased $(p<0.001)$. Doctors in the higher percentile and DSS groups tended to adopt the peer-comparison result to decrease their antibiotic prescriptions more than the non-DSS group.

\section{Discussion}

The study adopts a well-defined game theory to build a DSS and to embed the critical factors involved in coordination game equilibrium. It leverages an instrument for supporting doctors in evaluating prescription rates of antibiotics. The results of our analysis reveal that changes in doctors' prescriptions come after information about peer behavior is given and as a part of the prescribing decision process. A doctor's prescribing behavior can be improved by methods such as education, retrospective feedback, concurrent feedback, incentives, opinion leaders, patient education, and decision support systems (Ahluwalia et al., 1996; Dranove et al., 2003; Hellinger, 1996). One of these methods is peer-comparison feedback (i.e. doctor profiles), which provides data comparing the performance of one physician with that of his/her peers to improve provider accountability.

We focus on establishing a framework for pre-play communication so that the likelihood of a coordinated outcome or Pareto-efficient outcomes increases. After the quality report cards were disclosed, a significant decline in antibiotic prescriptions was detected, and the DSS group showed a greater decline than the non-DSS group at a different quarter $(p<0.001)$ in the higher percentile group. This result demonstrates that peer-comparison feedback with MCPA procedures as the monetary payoff in the coordination game motivated the doctors' practice change through interactive learning activities and competition with peers. In particular, doctors in the higher percentile group decreased their antibiotic prescriptions more when they used the DSS to get peercomparison feedback on prescribing patterns. Therefore, the use of pre-game communication is thus useful to the TBNHI authority, generating compliance significantly above physician education alone. These results are consistent with Wensing, Broge, Kaufmann-Kolle, Andres, and Szecsenyi (2004) study found that the

Table 4

The intergroup relationship at the baseline quarter $(n=1266)$.

\begin{tabular}{|c|c|c|c|c|c|c|}
\hline & \multicolumn{2}{|c|}{ Non-DSS group $(n=1102)$} & \multicolumn{2}{|c|}{ DSS group $(n=164)$} & \multirow[t]{2}{*}{$X^{2}$} & \multirow[t]{2}{*}{$p$-Value } \\
\hline & $N$ & $\%$ & $N$ & $\%$ & & \\
\hline \multicolumn{7}{|c|}{ Percentage of antibiotic prescriptions } \\
\hline Lower group & 561 & 88.6 & 72 & 11.4 & \multirow[t]{4}{*}{3.0267} & \multirow[t]{3}{*}{0.2202} \\
\hline Middle group & 268 & 84.8 & 48 & 15.2 & & \\
\hline Higher group & 273 & 86.1 & 44 & 13.9 & & \\
\hline \multicolumn{6}{|c|}{ MCPA procedures } & \\
\hline Non-accept & 328 & 85.9 & 54 & 14.1 & \multirow[t]{2}{*}{0.6684} & \multirow[t]{2}{*}{0.4136} \\
\hline Accept & 774 & 87.6 & 110 & 12.4 & & \\
\hline
\end{tabular}


Table 5

The relationship of the lower group at different quarters ( $n=633$ ).

\begin{tabular}{|c|c|c|c|c|c|c|}
\hline \multirow[t]{2}{*}{ Lower group } & \multicolumn{2}{|c|}{ Non-DSS group $(n=561)$} & \multicolumn{2}{|c|}{ DSS $\operatorname{group}(n=72)$} & \multirow[t]{2}{*}{$t$} & \multirow[t]{2}{*}{$p$-Value } \\
\hline & Mean & SD & Mean & SD & & \\
\hline Baseline I & 0.0393 & 0.0322 & 0.0430 & 0.0323 & -0.91 & 0.3648 \\
\hline Quarter I & 0.0419 & 0.0515 & 0.0407 & 0.0282 & 0.19 & 0.8469 \\
\hline Quarter II & 0.0425 & 0.0551 & 0.0377 & 0.0297 & 0.73 & 0.4676 \\
\hline Quarter III & 0.0471 & 0.0548 & 0.0447 & 0.0406 & 0.37 & 0.7132 \\
\hline Quarter IV & 0.0483 & 0.0569 & 0.0494 & 0.0481 & -0.15 & 0.8795 \\
\hline Quarter V & 0.0425 & 0.0584 & 0.0400 & 0.0392 & 0.35 & 0.7266 \\
\hline
\end{tabular}

Table 6

The relationship of the middle group at different quarters $(n=316)$.

\begin{tabular}{|c|c|c|c|c|c|c|}
\hline \multirow[t]{2}{*}{ Middle group } & \multicolumn{2}{|c|}{ Non- DSS group $(n=268)$} & \multicolumn{2}{|c|}{ DSS group $(n=48)$} & \multirow[t]{2}{*}{$t$} & \multirow[t]{2}{*}{$p$-Value } \\
\hline & Mean & SD & Mean & SD & & \\
\hline Baseline & 0.1785 & 0.0467 & 0.1943 & 0.0459 & -2.16 & 0.0313 \\
\hline Quarter I & 0.1664 & 0.0675 & 0.1795 & 0.0650 & -1.24 & 0.2149 \\
\hline Quarter II & 0.1681 & 0.0775 & 0.1812 & 0.0873 & -1.05 & 0.2923 \\
\hline Quarter III & 0.1648 & 0.0745 & 0.1734 & 0.0881 & -0.72 & 0.4744 \\
\hline Quarter IV & 0.1601 & 0.0793 & 0.1699 & 0.0859 & -0.79 & 0.4330 \\
\hline Quarter V & 0.1449 & 0.0805 & 0.1567 & 0.0877 & -0.92 & 0.3561 \\
\hline
\end{tabular}

Table 7

The relationship of the higher group at different quarters $(n=317)$.

\begin{tabular}{|c|c|c|c|c|c|c|}
\hline \multirow[t]{2}{*}{ Higher group } & \multicolumn{2}{|c|}{ Non-DSS group $(n=273)$} & \multicolumn{2}{|c|}{ DSS group $(n=44)$} & \multirow[t]{2}{*}{$t$} & \multirow[t]{2}{*}{$p$-Value } \\
\hline & Mean & SD & Mean & SD & & \\
\hline Baseline & 0.4333 & 0.1292 & 0.4147 & 0.1191 & 0.90 & 0.3711 \\
\hline Quarter I & 0.4042 & 0.1404 & 0.3248 & 0.1210 & 3.54 & 0.0005 \\
\hline Quarter II & 0.4015 & 0.1461 & 0.2971 & 0.1330 & 4.45 & 0.0000 \\
\hline Quarter III & 0.3797 & 0.1459 & 0.2809 & 0.1314 & 4.23 & 0.0000 \\
\hline Quarter IV & 0.3640 & 0.1440 & 0.2751 & 0.1335 & 3.84 & 0.0002 \\
\hline Quarter V & 0.3517 & 0.1526 & 0.2676 & 0.1396 & 3.43 & 0.0007 \\
\hline
\end{tabular}

application of quality circles had the intended effects on prescribing decisions in primary care in Germany.

Nevertheless, there are many factors that influence prescribing decisions in clinical practice, such as patient demand, the illness itself, information on drugs, health insurance payment regulation, pressure from colleagues and marketing by drug companies (Alvanzo et al., 2003; Chiang, 1997; Wensing et al., 2004). In particular, when doctors face the competition with peers that underlie the policy of patients having a free choice of physicians, this might provide an incentive to give in to patients' wishes for prescriptions (Wensing et al., 2004). Individual decision-making is based on a wide set of social factors, and egoism and altruism may also play a role in influencing decision-making (Jan, 2003; Kwon, 2006; Lin, Liu, Hsu, \& Wu, 2008). A rational decision maker does undeniably respond to economic incentives and tends to maximize his/ her monetary benefit (Hellinger, 1996). However, reimbursement by the TBNHI is subject to auditing strategies such as peer review activities or physician profile analyses to prevent false claims or over prescription. Hence, based on the financial realities, the doctors' patterns of prescribing will be affected by the policies of the third-party insurance payer. This is a general principal, widespread in the implementation of national health insurance-led systems in which essentially the payer decides what drugs are to be prescribed (Lambert et al., 1997). In the study of Mugford, Banfield and O'Hanlon (1991) argue that the feedback of statistical information, presented close to the time of decision-making, is likely to have a more direct effect on practice. A suitable time is important for policy maker who use the feedback loop to change practice patterns. Therefore, the DSS plays a critical role in the doctor's pre- scribing process and can enhance the ability of decision-makers by starting to consider their payoff and past decision-making behavior as part of the decision process (Farrell, 1993). This result verifies previous studies that have found that pre-play communication is an effective coordinating instrument in achieving Pareto-efficient outcomes (Brandts \& Charness, 2003).

In addition to these findings, we find that the DSS group was younger and had higher medical claim fees than non-DSS group. This indicates that the characteristics of individual doctors affect the collection of external information. It is especially important that a doctor is informed when he/she belong to "the higher percentage of antibiotic prescriptions" group or is in the "red light". Since doctors with higher medical claim fees have to face MCPA procedures, the monetary payoff affects them more than for doctors with lower medical claim fee. Many studies (Chinburapa et al., 1993; Denig et al., 1988) have also argued that the MCPA procedures are an auxiliary instrument used to change deviations in a doctor's patterns of prescribing and to decrease inappropriate drug prescriptions for the third-party insurance payer. As a result, publishing the audit rules of MCAP procedures often forces physicians to achieve high levels of compliance with drug prescribing regulations. This compliance will positively impact the doctors' patterns of prescribing and reduce health expenditures ( $\mathrm{Wu}$, Knill-Jones, Wilson, \& Craig, 2004). Meanwhile, applying peer-comparison feedback can enable doctors to benchmark their patterns of prescribing against these of their peers.

Nevertheless, the other important issue in the peer-comparison problem is how to define the relevant peer group? What biases are produced after disclosing the peer-comparison result? One result 
of defining peer groups may be the proverbial "slippery slope," with patients ultimately suffering (Kim et al., 1999). Shahian et al. (2001) indicated that one way the doctors can improve their apparent performance is by avoiding high-risk patients. Physicians will inevitably be concerned that treating such patients will lead to a poor outcome due to the negative quality rates. However, the disclosure of performance in health care seems sometimes to confuse the quality of indicators, and 'beating the system, not improving quality' may pervert behavior and engender a defensive culture detrimental to quality (Mason \& Street, 2005). Therefore, we need to use the appropriate information with performance assessments for overlooking this bias and take account of known confounding variables.

\section{Conclusions and suggestions for future research}

We employ a DSS with coordinating instruments for doctors to integrate relevant information into their prescribing decision process. Our study shows that game-theory modeling, built into the decision support systems, has made significant contributions to understanding the coordinating instruments for managing the doctors' practice patterns and encouraging doctors toward these patterns favored by the third-party insurance payer. Herein, the real-world data set, gathered from the NHI program in Taiwan, shows that doctors' patterns of prescribing can be reshaped through peer-comparison and the monetary payoff of MCPA procedures. Our study also shows that the quality report cards help the doctor filter and evaluate information for establishing suitable patterns in their practice management. In the future, integrating electronic clinic data with practice management data on the doctor practices, doctors will easily be able to compare their patterns of practice with their peers, and thus control health costs as well as increase efficiency.

Some limitations of this study should be mentioned. First, despite significant efforts to reshape doctors' prescribing behaviors, many doctors were not involved in our sample. Therefore our study is an uncontrolled before and after comparison because use of the DSS was voluntary and this is a natural experiment that did not control different case mixes of disease distribution across doctors. Potential biases exist as the results are likely to be existed toward better-known report card efforts. Second, regardless of our extensive follow-up efforts, we limited the patterns of prescribing antibiotics information to claim data, and medical records did not confirm this, so the patterns of prescribing antibiotics bias cannot be ascertained. It may be that the percentages of antibiotics prescribed are different, or perhaps the percentages are not accurately submitted in the claim data. Nevertheless, this study concludes that disclosing the peer-comparison information via DSS is a beneficial coordinating instrument to aid doctors' practice compliance with drug prescription regulations.

This research has two important policy implications. First, while the promise on the quality of care is voluntary for doctors, if policymakers can facilitate a form of pre-play communication the among doctors before the claim auditing process it will be increase the compliance of doctors. Notably, when the health care operates in a management-oriented system, doctors' actions are highly reliant on the perception of their monetary payoff and the cognitive experience of the clinical care. Therefore, a DSS is used as the coordinating instrument for the disclosure of quality report cards can act as a good facilitator to push doctors toward a better quality patient care. Second, policy-makers can acquire more knowledge about applying behavioral game modeling into designing a decision support system, and stimulate a positive attitude in the players (health care providers) for improving cost savings based on the prescription patterns.
Furthermore, this study encouraged the TBNHI to expand its disclosure of quality report cards and peer-comparison results on its website. These results were highlighted to attract physicians and beneficiaries concerned about the related indicators of quality care in the NHI. Other countries may also find our study results helpful when investigating the issue of prescriptions as well as cross-country comparisons.

Opportunities for further research are abundant. First, further research should examine not only variations in antibiotics prescription by different specialists, but also building a suitable mechanism that will reflect various diseases with certain antibiotics. Second, there is need for infrastructure and technical assistance to facilitate development of the peer-comparison process and the sharing of information among doctors. Greater systematization may be required, and the goal should be to enhance the doctors' involvement. Third, a plurality of communication approach and research questions in evaluation is needed so as to broaden our understanding of doctors' prescribing behavior and sue of DSS applications. To this end, the buildings of an intelligent DSS to detect and measure changes in doctors' prescription rates of antibiotics for diagnosing abnormal practices are highly necessary and useful for practitioners. We hope that many researchers will employ DSS approaches and game theory modeling for further investigation of this important area.

\section{Acknowledgements}

The authors would like to thank Chih-Lin Fang, Lu Tsai, MaoLong Chen and the Bureau of National Health Insurance of the Southern Region of Taiwan, for their helping in providing the research database and creating the DSS. The referees are also appreciated for their valuable comments and criticisms.

\section{References}

Ahluwalia, J. S., Weisenberger, M. L., Bernard, A. M., \& McNagny, S. E. (1996). Changing physician prescribing behavior: A low-cost administrative policy that reduced the use of brand name nonsteroidal anti-inflammatory drugs. Preventive Medicine, 25, 668-672.

Alm, J., \& McKee, M. (2004). Tax compliance as a coordination game. Journal of Economic Behavior E' Organization, 54(3), 297-312.

Alvanzo, A. H., Cohen, G. M., \& Nettleman, M. (2003). Changing physician behavior: Half-empty or half-full? Clinical Governance, 8(1), 69-78.

Berndt, D. J., Hevner, A. R., \& Studnicki, J. (2003). The Catch data warehouse: Support for community health care decision-making. Decision Support Systems, 35, 367-384.

Bhargava, H. K., Power, D. J., \& Sun, D. (2007). Progress in Web-based decision support technologies. Decision Support Systems, 43(4), 1083-1095.

Bradley, C. P. (1991). Decision making and prescribing patterns: A literature review. Family Practice, 8, 2762-2787.

Brandts, J., \& Charness, G. (2003). Truth or consequences: An experiment. Management Science, 49(1), 116-130.

Brownell, M. D., Roos, N. P., \& Roos, R. L. (2001). Monitoring health reform: A report card approach. Social Science \&' Medicine, 52, 657-670.

Burton, A., \& Sefton, M. (2004). Risk, pre-play communication and equilibrium. Games and Economic Behavior, 46, 23-40.

Camerer, C. F. (2003). Behavioral game theory: Experiments in strategic interaction. Princeton, NJ: Princeton University Press.

Cheng, T. M. (2003). Taiwan's new national health insurance program: Genesis and experience so far. Health Affairs, 22(3), 61-76.

Chiang, T. L. (1997). Taiwan's 1995 health care reform. Health Policy, 39, 225-239.

Chinburapa, V. et al. (1993). Physician prescribing decisions: The effects of situational involvement and task complexity on information acquisition and decision-making. Social Science \& Medicine, 1993, 1473-1482.

Colman, A. M. (1995). Game theory and its applications in the social and biological sciences (2nd ed.). Oxford, Boston: Butterworth-Heinemann.

Cooper, R. et al. (1990). Selection criteria in coordination games: Some experimental results. American Economic Review, 80, 218-233.

Cooper, R. et al. (1992). Communication in coordination games. Quarterly Journal of Economics, 53, 739-771.

Denig, P., Haaijer-Ruskamp, F. M., \& Zijsling, D. H. (1988). How physicians choose drugs. Social Science E Medicine, 27, 1381-1386.

Dixit, A., \& Skeath, S. (1999). Games of strategy. New York: WW Norton and Company. 
Dranove, D., Kessler, D., McClellan, M., \& Satterthwaite, M. (2003). Is more information better? The effects of "report cards" on health care providers. Journal of Political Economy, 111(3), 555-588.

Elgood, C. (1984). Handbook of management games (3rd ed.). Hants, England: Aldershot.

Ess, S. M., Schneeweiss, S., \& Szucs, T. D. (2003). European healthcare policies for controlling drug expenditure. PharmacoEconomics, 21(2), 89-103.

Farrell, J. (1993). Meaning and credibility in cheap-talk games. Games Economic Behavior, 5, 514-531.

Ford, E. W., Wells, R., \& Bailey, B. (2004). Sustainable network advantages: A game theoretic approach to community-based health care coalition. Health Care Management Review, 29(2), 159-169.

Fu, H. H., Tsai, H. T., Lin, C. W., \& Wei, D. (2004). Application of a single sampling plan for auditing medical-claim payments made by Taiwan National Health Insurance. Health Policy, 70(2), 185-195.

Hellinger, F. J. (1996). The impact of financial incentives on physician behavior in managed care plans: A review of the evidence. Medical Care Research and Review, 53(3), 294-314.

Hibbard, J. H., Stockared, J., \& Tusler, M. (2003). Does publicizing hospital performance stimulate quality improvement efforts? Health Affairs, 22(2), 84-94.

Hibbard, J. H., Stockared, J., \& Tusler, M. (2005). Hospital performance reports: Impact on quality, market share and reputation. Health Affairs, 24(4), 1150-1161.

Jaegher, K. D., \& Jegers, M. (2001). The physician-patient relationship as a game of strategic information transmission. Health Economics, 10, 651-668.

Jan, S. (2003). A perspective on the analysis of credible commitment and myopia in health sector decision making. Health Policy, 63(1), 269-278.

Kim, C. S. et al. (1999). Physician education and report cards: do they make the grade? Results from a randomized controlled trial. The American Journal of Medicine, 107(6), 556-560

Kim, T. H., Hong, G. H., \& Park, S. C. (2008). Developing an intelligent web information system for minimizing information gap in government agencies and public institutions. Expert Systems with Applications, 34, 1618-1629.

Kruck, S. E., Teer, F., \& Christian, W. A. (2008). GSLAP: A graph-based web analysis tool. Industrial Management \& Data Systems, 108(2), 162-172.

Kwon, O. (2006). The potential roles of context-aware computing technology in optimization-based intelligent decision-making. Expert Systems with Applications, 31, 421-629.

Lambert, B. L. et al. (1997). Factors associated with antibiotic prescribing in a managed care setting: An exploratory investigation. Social Science $\mathcal{E}$ Medicine, 45(12), 1767-1779.

Lee, K. C., \& Chung, N. (2005). A Web DSS approach to building an intelligent internet shopping mall by integrating virtual reality and avatar. Expert Systems with Applications, 28, 333-346.

Liang, H., Xue, Y., \& Berger, B. A. (2006). Web-based intervention support system for health promotion. Decision Support Systems, 42(1), 435-449.

Lin, C., Liu, A. C. P., Hsu, M. L., \& Wu, J. C. (2008). Pursuing excellence in firm core knowledge through intelligent group decision support system. Industrial Management \& Data Systems, 108(3), 277-296.

Lovell, N. H., \& Celler, B. G. (1999). Information technology in primary health care International Journal of Medical Informatics, 55(1), 9-22.

March, S. T., \& Hevner, A. R. (2007). Integrated decision support systems: a data warehousing perspective. Decision Support Systems, 43(3), 1031-1043.

Maryniuk, G. A. (1990). Practice variation: Learned and socio-economic factors. Advances Dental Research, 4, 19-24.

Mason, A., \& Street, A. (2005). Publishing outcome data: Is it an effective approach? Journal of Evaluation in Clinical Practice, 12(1), 37-48.
McNamara, P. (2006). Provider-specific report cards: A tool for health sector accountability in developing countries. Health Policy and Planning, 21(2), 101-109.

Mugford, M., Banfield, P., \& O'Hanlon, M. (1991). Effects of feedback of information on clinical practice: A review. British Medical Journal, 303(17), 398-402.

Nemati, H. R., Steiger, D. M., Iyer, L. S., \& Herschel, R. T. (2002). Knowledge warehouse: An architectural integration of knowledge management, decision support, artificial intelligence and data warehousing. Decision Support Systems, 33, 143-161.

O'Brien, B. J. (1988). A game-theoretic approach to donor kidney sharing. Social Science and Medicine, 26, 1109-1116.

Okunade, A. A., \& Suraratdecha, C. (2006). The pervasiveness of pharmaceutical expenditure inertia in the OECD countries. Social Science Medicine, 63(1), 225-238.

Pedersen, M. K., \& Larsen, M. H. (2001). Distributed knowledge management based on product state models - The case of decision support in health care administration. Decision Support Systems, 31, 139-158.

Rasmusen, E. (1994). Games and information: An introduction to game theory (2nd ed.). Cambridge, MA: Blackwell.

Ruland, C. M., \& Bakken, S. (2002). Developing, implementing, and evaluating decision support systems for shared decision making in patient care: A conceptual model and case illustration. Journal of Biomedical Informatics, 35(5-6), 313-321.

Schelling, T. C. (1960). The strategy of conflict. Cambridge, MA: Harvard University Press.

Shahian, D. et al. (2001). Cardiac surgery report cards: Comprehensive review and statistical critique. Annals of Thoracic Surgery, 72, 2155-2168.

Shiell, A., \& Chapman, S. (2000). The inertia of self-regulation: A game-theoretic approach to reducing passive smoking in restaurants. Social Science E Medicine, 51, 1111-1119.

Short, D., Frischer, M., \& Bashford, J. (2004). Barriers to the adoption of computerized decision support systems in general practice consultations: a qualitative study of GPs' perspectives. International Journal of Medical Informatics, 37(4), 257-362.

Sintchenko, V., \& Coiera, E. W. (2003). Which clinical decisions benefit from automation? A task complexity approach. International Journal of Medical Informatics, 70(2-3), 309-316.

Slovensky, D. J., Fottler, M. D., \& Houser, H. W. (1998). Developing an outcomes report card for hospitals: A case study and implementation guideline. Journal of Healthcare Management, 43(1), 15-30.

Tremblay, M. C., Fuller, R., Berndt, D., \& Studnicki, J. (2007). Doing more with more information: changing healthcare planning with OLAP tools. Decision Support Systems, 43(4), 1305-1320.

Tsai, W. H., \& Kuo, H. C. (2007). The Internet and healthcare in Taiwan: Value-added applications on the medical network in the National Health Insurance smart card system. International Journal of Electronic Healthcare, 3(4), 417-432.

Van Huyck, J. B., Battalio, R. C., \& Beil, R. (1990). Tacit coordination games, strategic uncertainty and coordination failure. American Economic Review, 80, 234-248.

Watson, H. J., Fuller, C., \& Ariyachandra, T. (2004). Data warehouse governance. Best practices at Blue Cross and Blue Shield of North Carolina. Decision Support Systems, 38, 435-450.

Wensing, M., Broge, B., Kaufmann-Kolle, P., Andres, E., \& Szecsenyi, J. (2004). Quality circles to improve prescribing patterns in primary medical care: what is their actual impact? Journal of Evaluation in Clinical Practice, 10(3), 457-466.

Wu, O., Knill-Jones, R., Wilson, P., \& Craig, N. (2004). The impact of economic information on medical decision making in primary care. Journal of Evaluation in Clinical Practice, 10(3), 407-411. 